\title{
Effects of Ivabradine on Hemodynamic and Functional Parameters in Left Ventricular Systolic Dysfunction: a Systematic Review and Meta-analysis
}

\author{
Peysh A. Patel, MA' , Noman Ali, PhD ${ }^{7}$, Ashwin Roy, BSc ${ }^{2}$, Stuart Pinder, MSc ${ }^{7}$, \\ Richard M. Cubbon, $P h D^{7}$, Mark T. Kearney, $M D^{7}$, and Klaus K. Witte, $M D^{7}$ \\ 'Department of Cardiology, Leeds General Infirmary, Leeds, UK; ²Department of Cardiology, Tameside General Hospital, Greater Manchester, UK.
}

\begin{abstract}
BACKGROUND: Ivabradine is licensed as add-on therapy in patients with severe left ventricular systolic dysfunction (LVSD), normal sinus rhythm, and suboptimal heart rate (HR) control, but effects are not fully established. This study sought to assess the impact of ivabradine therapy on hemodynamic and functional outcome measures in all patients with LVSD.
\end{abstract}

METHODS: MEDLINE (1996-2017), Embase (19962017), Cochrane Central Register of Controlled Trials (CENTRAL), Cochrane Database of Systematic Reviews, ClinicalTrials.gov, and ISI Web of Science were searched for randomized clinical trials (RCTs) comparing standard medical therapy (SMT) plus ivabradine to SMT alone for patients with LVSD of any severity. Each trial was assessed using the Cochrane Collaborations Risk of Bias tool.

RESULTS: Eight RCTs with 17,823 patients were included. Add-on use of ivabradine reduced resting HR (mean difference [MD] $10.3 \mathrm{bpm} ; p<0.001)$, improved ejection fraction (EF) (MD 3.6\%, $p<0.001)$, and preserved systolic blood pressure (MD $3.4 \mathrm{mmHg} p=0.09$ ). Stratified analyses according to severity of LVSD did not influence conferred benefits on HR and EF. Small improvements were noted in exercise tolerance (standardized MD $5.9 \mathrm{~s} ; p=$ 0.004 ) and peak oxygen consumption (MD $2.9 \mathrm{ml} / \mathrm{kg} /$ $\min ; p=0.02$ ).

DISCUSSION: Adjunct therapy with ivabradine in patients with LVSD results in a favorable hemodynamic profile and correlates with improved functional capacity. Benefits appear to be broadly preserved irrespective of baseline EF. This was a meta-analysis of RCTs, though limited by exclusion of post hoc analyses, lack of access to patient level data, and inter-study variability in some baseline characteristics. Further, large-scale RCTs are warranted to evaluate effectiveness of ivabradine in cohorts with non-severe LVSD.

KEY WORDS: ivabradine; left ventricular systolic dysfunction; systematic review; meta-analysis.

J Gen Intern Med 33(9):1561-70

DOI: $10.1007 / \mathrm{s} 11606-018-4578-4$

(c) Society of General Internal Medicine 2018

Peysh A. Patel and Noman Ali have joint authorship.

Received April 13, 2018

Revised June 5, 2018

Accepted July 2, 2018

Published online July 18, 2018

\section{INTRODUCTION}

Chronic heart failure (CHF) due to left ventricular systolic dysfunction (LVSD) affects over 23 million people worldwide and constitutes $1-2 \%$ of total health care expenditure. ${ }^{1}$ Recent trends are indicative of worsening prevalence and commensurate economic burden, mostly attributable to an aging population and improved survival after ischemic heart disease (IHD). There has been a paradigm shift in the management of LVSD, primarily influenced by advancements in pharmacotherapy. ${ }^{2}$ Angiotensin-converting enzyme (ACE) inhibitors or angiotensin receptor blockers (ARB), aldosterone antagonists, and, most recently, angiotensin receptor-neprilysin inhibitors (ARNI) have all demonstrated significant benefit on outcomes, mediated in part through modulation of the reninangiotensin-aldosterone (RAA) neurohormonal axis. ${ }^{3-5}$

Complementary use of beta-blockers (BB) has also exerted positive influence in abrogating symptom burden and improving prognosis. ${ }^{6}$ In addition to neurohormonal regulation, BB have negative chronotropic effects which increase diastolic perfusion time and reduce myocardial demand. Elevated resting heart rate (HR) appears to be a pathophysiological contributor in LVSD. ${ }^{7}$ In patients with acute decompensated heart failure, $\mathrm{HR} \geq 70 \mathrm{bpm}$ in normal sinus rhythm (NSR) is predictive of in-hospital mortality and the resting HR upon discharge influences 12-month hospital readmission and mortality rates. ${ }^{8,9}$ As such, the negative chronotropic effects of BB are postulated to be at least partly responsible for observed benefits. However, BB also confer negative inotropy and lower blood pressure (BP), limiting their use in patients with marked or symptomatic hypotension.

Ivabradine is a relatively novel agent that specifically inhibits hyperpolarization-activated cyclic nucleotide-gated ( $\mathrm{HCN})$ channels, thereby decreasing the pacemaker current (If ) within the sinoatrial node. ${ }^{10}$ This reduces HR without modifying inotropy, lusitropy and intracardiac conduction. It therefore offers the potential for additional HR lowering in those that poorly tolerate $\mathrm{BB}$ or have suboptimal rate control despite use.

The Systolic Heart failure treatment with the I(F) inhibitor ivabradine Trial (SHIFT) demonstrated that in patients with stable, severe LVSD and a HR $\geq 70 \mathrm{bpm}$ at baseline despite maximally tolerated BB dose, selective administration of ivabradine reduced heart failure hospitalization and cardiovascular 
death. ${ }^{11}$ Whilst this landmark study highlighted potential benefit, it was noted in subgroup analyses that a significant treatment effect was only observed in patients with a higher $\mathrm{HR}$ at baseline. Current American Heart Association (AHA) and European Society of Cardiology (ESC) guidelines advocate therapy (class IIa indication) for those with symptomatic, severe LVSD, on standard medical therapy (SMT) and in NSR but with resting HR $\geq 70 \mathrm{bpm} .{ }^{12,13}$

The purpose of this study was to conduct a systematic review and meta-analysis of randomized clinical trials (RCTs) to further assess the impact of add-on therapy with ivabradine on hemodynamic parameters and functional outcomes in patients with LVSD of any severity and symptomatic class.

\section{METHODS}

\section{Data sources and searches}

MEDLINE (inception to 3 August 2017), Embase (inception to 3 August 2017), Cochrane Central Register of Controlled Trials (CENTRAL), Cochrane Database of Systematic Reviews, ClinicalTrials.gov, and ISI Web of Science were searched using a priori database-appropriate MESH terms relating to ivabradine, LVSD, and RCTs. Additional studies were sought using WorldCat database and Google Scholar, with derived references from these sources used to seek other potentially relevant citations. Two authors (PAP, NA) independently performed electronic searches of all databases. From this initial search, duplicate articles were removed and the remainder screened for suitability based on title and abstract. A third author (KKW) aided with adjudication in instances where a consensus could not be reached.

\section{Study selection}

Studies were eligible if they included participants with objective evidence of LVSD, defined using an established imaging modality and quantified as left ventricular ejection fraction (LVEF) $<55 \%$. LVSD of any etiology and New York Heart Association (NYHA) functional class was considered, though patients had to be optimized on SMT. Only RCTs with ivabradine as the intervention were deemed suitable, with consideration of all doses and uptitration regimes. Pre-specified primary outcomes were hemodynamic parameters, namely HR, BP, and ejection fraction (EF). Secondary outcomes included plasma N-terminal-pro hormone B-type natriuretic peptide (NT-proBNP) levels, exercise tolerance, peak oxygen consumption, NYHA class, and safety profile. All studies published in English language were considered, but subgroup and post hoc analyses were excluded.

\section{Data extraction and quality assessment}

Data extraction was performed using a pre-specified template constructed on Microsoft Excel 2016. This was split and conducted independently by two authors (AR, SP), with subsequent verification by two separate authors (PAP, NA) to ensure validity and accuracy. Relevant information pertaining to baseline participant characteristics, inclusion and exclusion criteria, interventions, and summary outcome measures was collated. For studies in which patients were evaluated at multiple timepoints, the shortest follow-up duration was selected to be broadly consistent with the other trials. Similarly, if there were multiple intervention groups, the highest dose of ivabradine was selected for comparison purposes. The quality of each trial was assessed independently by two authors (AR, SP) using the Cochrane Collaborations Risk of Bias tool. ${ }^{14} \mathrm{~A}$ third author (PAP) was consulted to resolve any discrepancies in interpretation.

\section{Data synthesis and analysis}

This was performed concurrently by two authors (PAP, NA) using RevMan 5.3 software (Version 5.3.5, 2014). All data were processed in accordance with the PRISMA statement. ${ }^{15}$ PAP had full access to all the data in the study and takes responsibility for its integrity and the data analysis. Continuous data are presented as means, unless otherwise stated, and categorical data as percentages. Groups were compared using unpaired Student's $t$ tests for normally distributed continuous data, and Pearson's $\chi^{2}$ tests with Yates' correction for categorical data. All tests were two-sided with statistical significance defined by $p$ values $<0.05$.

Dichotomous outcome data were analyzed using the Mantel-Haenszel approach and expressed as risk ratios (RR). Continuous outcome data were assessed using mean difference (MD) or standardized mean difference (SMD), depending on whether measurement scales were consistent between studies. An inverse variance method was adopted, with an intention-to-treat (ITT) principle for all reported outcome measures. The pooled comparisons of control (SMT) and intervention (SMT + ivabradine) groups were depicted visually using forest plots. All effect estimates derived from metaanalyses were provided using $95 \%$ confidence intervals, with $p$ values $<0.05$ considered statistically significant. The impact of statistical heterogeneity on this estimate was quantified using the $I^{2}$ test, with categorization defined as low $(<25 \%)$, moderate $(25-50 \%)$ or substantial $(>50 \%)$. A random effects model was applied as default to facilitate presence of natural heterogeneity between studies. Funnel plots were not used to report publication bias in view of low total number of studies $(<10)$. To assess robustness of findings, a sensitivity analysis for select outcomes was conducted with exclusion of the study of maximal weighting.

\section{RESULTS}

\section{Study Selection}

Initial database searches and identification through other sources yielded 316 studies, of which 294 were eligible following 
removal of duplicates. Of these, 240 were excluded after screening based upon title and abstract. This resulted in 54 full-text articles which were individually assessed. Eight of these studies (2.7\% of those initially eligible) were deemed suitable for qualitative and quantitative analysis. ${ }^{11,16-22} \mathrm{An}$ overview of the study selection process is depicted using a PRISMA flow diagram (see Fig. 1).

\section{Participant Characteristics}

The included studies were undertaken between 2008 and 2015, with 17,823 patients in total. An overview of study designs is provided in Table 1. Three were multi-country, with the largest including 10,917 patients from 781 centers across 33 countries. ${ }^{17}$ Baseline characteristics for individual trials are detailed in Table 2. In total, there were 8895 patients in the control arm and 8928 patients in the intervention arm. Pooled, weighted characteristics at baseline were well matched (Table 3). Mean age was 63 years, with equivalent prevalence

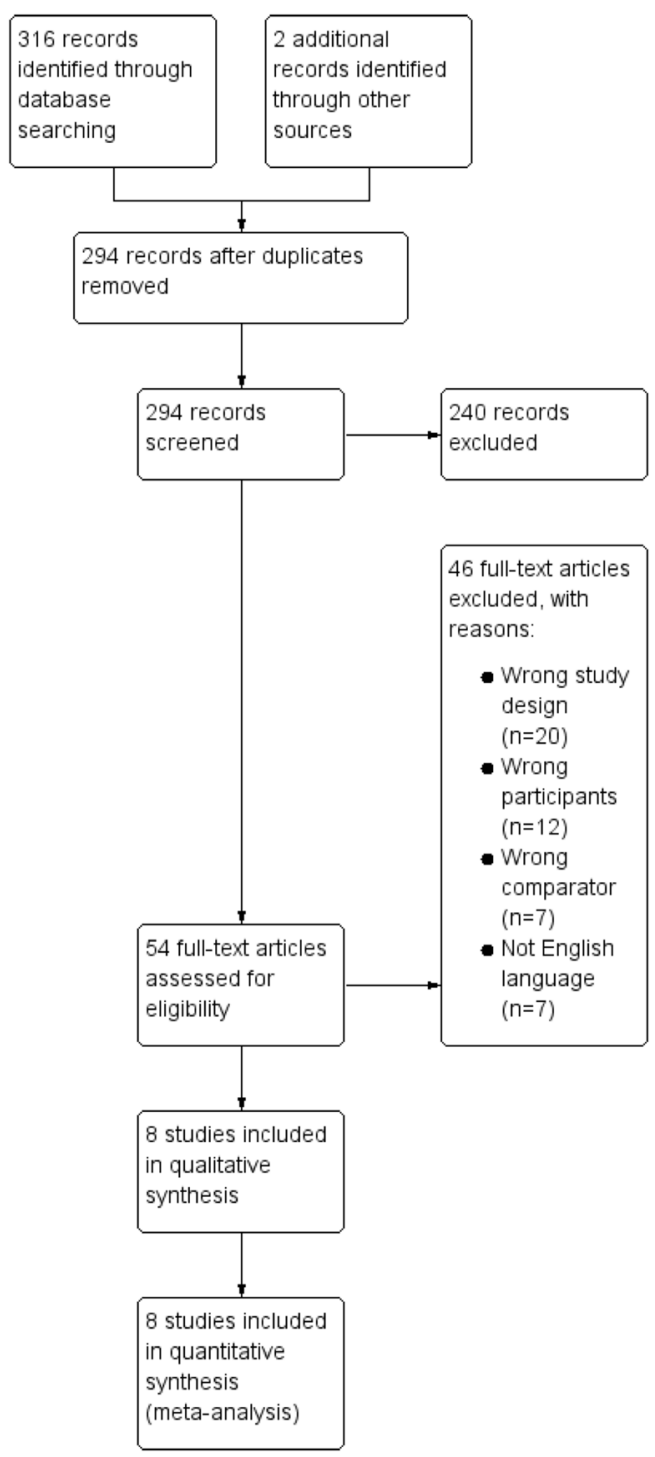

Figure 1 PRISMA study flow diagram. of hypertension (69\%), diabetes mellitus (35\%), and prior IHD (76\%). Physiological parameters including resting HR, BP, and EF were also nearly identical between the control and ivabradine groups.

\section{Risk of Bias}

A summary of judgements on methodological quality based on pre-specified criteria is provided in Fig. 2 and listed for each individual study. Sequence generation was not described in four

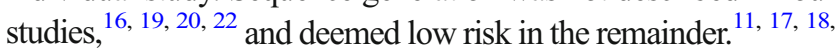
${ }^{21}$ Allocation concealment was unreported in five studies. ${ }^{16,18-}$ 20, 22 Adequate blinding of participants, personnel, and outcome assessors was achieved in four studies. ${ }^{11,17,20,21}$ Attrition bias was deemed low risk in all eight studies. Selective reporting was considered low-risk in six studies ${ }^{11,16}{ }^{19,21}$ and unclear in the remaining two. ${ }^{20,22}$ The authors of three studies ${ }^{11,17,21}$ declared industry sponsorship. Overall, all studies were deemed suitable for systematic review and meta-analyses, where appropriate.

\section{Hemodynamic Parameters}

Heart Rate. Change in resting HR from baseline was reported in all eight included studies $(n=17,823)$, with a median follow-up duration of 3 months. Overall, there was a reduction in those treated with SMT + ivabradine as opposed to SMT alone with a mean difference (MD) of $10.3 \mathrm{bpm}$ (Fig. 3a) (95\% CI 7.8-12.8; $p<0.001)$. However, statistical inter-study heterogeneity was substantial $\left(I^{2}=99 \% ; p<0.001\right)$. A sensitivity analysis with exclusion of the study of largest weighting $(n=10,917)$ preserved the effect estimate (MD $10.8 \mathrm{bpm}$, 95\% CI 7.9-13.7; $p<0.001$ ) (Fig. 3b).

Blood Pressure. An assessment of change in systolic BP was performed in three studies $(n=169),{ }^{16}, 20,22$ with a median follow-up period of 3 months. No difference was observed (MD $3.4 \mathrm{mmHg}$, 95\% CI $-0.5-7.3 ; p=0.09$ ), with substantial heterogeneity $\left(I^{2}=94 \% ; p<0.001\right)$ (Fig. 4a). Data on change in diastolic BP was available from two studies $(n=109),{ }^{16,22}$ with a median follow-up of 2.5 months. A small but statistically significant MD of $4.2 \mathrm{mmHg}(95 \%$ CI $3.1-5.3 ; p<0.001)$ with low heterogeneity $\left(I^{2}=15 \% ; p=0.28\right)$ was observed (Fig. $\left.4 \mathrm{~b}\right)$.

Ejection Fraction. Change in EF was quantified in five studies, but one $\mathrm{e}^{22}$ did not present the raw data and as such, four studies were eligible for inclusion $(n=223){ }^{16,18,20,21}$ Median follow-up duration was 2.5 months. There was a small but significant improvement in EF in the SMT + ivabradine group, with a MD of $3.6 \%$ (95\% CI 2.4-4.8; $p<0.001$ ) (Fig. 5a). Heterogeneity appeared to be substantial $\left(I^{2}=\right.$ $75 \% ; p=0.007)$, but the overall trend was preserved when the study of largest weighting $(n=81)$ was eliminated (MD $3.5 \%, 95 \%$ CI $1.2-5.7 ; p=0.003$ ) (Fig. 5b).

NT-proBNP. Three studies compared NT-proBNP levels $(n=$ 239), ${ }^{19}{ }^{21}$ with a median follow-up of 3 months. In the SMT + 
Table 1 Study Designs

\begin{tabular}{|c|c|c|c|c|c|}
\hline \multirow[t]{2}{*}{$\overline{\overline{\text { Study ID }}}$} & \multirow[t]{2}{*}{$\overline{\text { Year }}$} & \multirow{2}{*}{$\begin{array}{l}\text { Centers } \\
\text { (by country) }\end{array}$} & \multirow[t]{2}{*}{ Inclusion criteria } & \multicolumn{2}{|c|}{ 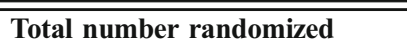 } \\
\hline & & & & $\begin{array}{l}\text { SMT } \\
{[n=8895]}\end{array}$ & $\begin{array}{l}\text { SMT + ivabradine } \\
{[n=8928]}\end{array}$ \\
\hline Amosova et al. & 2011 & Italy; Ukraine & $\begin{array}{l}\text { History of MI, LVEF }<45 \% \text {; CCS class I/II; NSR with resting } \\
\text { HR }>60 \text { bpm; SMT for } \geq 3 \text { months }\end{array}$ & 12 & 17 \\
\hline Fox et al. & 2008 & Multiple (33) & $\begin{array}{l}\text { Age }>55 \text { (or }>18 \text { if DM); CAD; LVEF }<40 \% \text {; NYHA } \\
\text { class I/II/III; NSR with resting HR }>60 \mathrm{bpm} \text {; stable symptoms } \\
\text { for } \geq 3 \text { months and SMT for } \geq 1 \text { month }\end{array}$ & 5438 & 5479 \\
\hline Mansour et al. & 2011 & Egypt & $\begin{array}{l}\text { Idiopathic DCM; LVEF }<40 \% \text {; NYHA class III/IV; NSR } \\
\text { with resting HR }>70 \text { bpm; SMT for } \geq 1 \text { month }\end{array}$ & 23 & 30 \\
\hline Ordu et al. & 2015 & Turkey & $\begin{array}{l}\text { LVEF }<35 \% \text {; NYHA class I/II; NSR with resting HR }>70 \mathrm{bpm} \text {; } \\
\text { SMT (duration not specified); previous hospital admission } \\
\text { for CHF within } 12 \text { months }\end{array}$ & 49 & 49 \\
\hline Sarullo et al. & 2010 & Italy & $\begin{array}{l}\text { Ischemic CHF; LVEF } \leq 40 \% \text {; NYHA class II/III, NSR with } \\
\text { resting HR }>70 \text { bpm; SMT for } \geq 3 \text { months }\end{array}$ & 30 & 30 \\
\hline Swedberg et al. & 2010 & Multiple (37) & $\begin{array}{l}\text { Age }>18 \text {; all etiologies aside from CHD or primary severe valve } \\
\text { disease; LVEF } \leq 35 \% \text {; NYHA class II/III/IV; NSR with } \\
\text { HR }>70 \text { bpm; stable symptoms and SMT for } \geq 1 \text { month; } \\
\text { previous hospital admission for CHF within } 12 \text { months }\end{array}$ & 3264 & 3241 \\
\hline Tsutsui et al. & 2016 & Japan & $\begin{array}{l}\text { Age } \geq 20 \text { years; LVEF } \leq 35 \% ; \text { NYHA I/II; NSR with resting } \\
\text { HR } \geq 75 \text { bpm; SMT (duration not specified) }\end{array}$ & 41 & 40 \\
\hline Volterrani et al. & 2011 & Italy & $\begin{array}{l}\text { Aged } 18-90 \text { years; diagnosis of CHF for } \geq 12 \text { months (severity } \\
\text { not specified); NYHA II/III; SMT (duration not specified); } \\
\text { stable symptoms for } \geq 3 \text { weeks }\end{array}$ & 38 & 42 \\
\hline
\end{tabular}

CAD coronary artery disease, CCS Canadian Cardiovascular Society, CHD congenital heart disease, CHF congestive heart failure, DCM dilated cardiomyopathy, DM diabetes mellitus, HR heart rate, LVEF left ventricular ejection fraction, MI myocardial infarction, NSR normal sinus rhythm, NYHA New York Heart Association, SMT standard medical therapy

ivabradine group, there was a trend towards reduction with MD of $462.9 \mathrm{pg} / \mathrm{ml}(95 \%$ CI $9.5-916.3 ; p=0.05)$. Heterogeneity was substantial $\left(I^{2}=98 \% ; p<0.001\right)$.

\section{Functional Capacity}

Exercise Tolerance. Four studies assessed exercise tolerance, but one was excluded from meta-analysis on the basis that data were presented as 6-min walk distance (6MWD) rather than maximum duration of exercise. ${ }^{22}$ Median follow-up period of the three remaining studies $(n=142)^{16,18,20}$ was 3 months, with quantification using a treadmill or cycle ergometer. A marginal improvement in exercise tolerance was observed in the SMT + ivabradine group, with a SMD of $5.9 \mathrm{~s}(95 \%$ CI 1.9-10.0; $p=$ $0.004)$ and substantial heterogeneity $\left(I^{2}=98 \% ; p<0.001\right)$.

Peak Oxygen Consumption. Two studies detailed assessment of peak oxygen consumption $(n=140),{ }^{20,} 22$ with a median follow-up of 3 months. Both utilized a cycle ergometer for exercise testing. An improvement in peak consumption was detected in the SMT + ivabradine group, with a MD of $2.9 \mathrm{ml} /$ $\mathrm{kg} / \mathrm{min}(95 \%$ CI $0.6-5.3 ; p=0.02)$. Heterogeneity was quantified to be substantial $\left(I^{2}=99 \% ; p<0.001\right)$.

NYHA Functional Class. Five studies assessed differences in NYHA functional class. Meta-analysis was not performed due to significant inter-study variability in reporting of outcomes. One study did not present the original dataset ${ }^{21}$, but stated that "class did not change". The largest study $(n=6506){ }^{11}$ noted a small but significant difference in the proportion that improved their functional class $(28 \%$ [SMT + ivabradine] vs $24 \%$ [SMT alone]; $p=0.001$ ), and this correlated with trends observed in smaller studies at 3 months ${ }^{18,22}$ and 6 months. ${ }^{19}$
Quality of Life. Effects on quality of life (QoL) were reported in three studies $(n=193)$, with two using the Minnesota Living With Heart Failure (MLWHF) scale ${ }^{18,}$ 20 and the other applying a visual analogue scale. ${ }^{22}$ There was a trend towards improvement in QoL score in the SMT + ivabradine group (SMD of 7.0, 95\% CI $0.2-14.1 ; p=0.06)$, though heterogeneity was substantial $\left(I^{2}=99 \% ; p<0.001\right)$.

\section{Safety Profile}

Three studies detailed tolerability of ivabradine. In the largest of these $(n=10,917),{ }^{17}$ discontinuation rates were $28 \%$ (SMT + ivabradine) vs $16 \%$ (SMT alone). The primary reason was bradycardia, though notably, only $21 \%$ of the ivabradine subgroup were symptomatic. More rarely, visual symptoms such as blurred vision and phosphenes were implicated and resolved after drug termination. Overall incidence of serious adverse events was equivalent between the ivabradine and control groups (23 vs $23 \% ; p=0.70)$.

A second study $(n=6505)^{11}$ documented $2 \%$ higher withdrawal rates in the SMT + ivabradine group (21 vs $19 \% ; p=$ $0.02)$, though serious adverse events occurred with lower frequency ( 45 vs $48 \% ; p=0.03$ ). Although both symptomatic ( 5 vs $1 \% ; p<0.001)$ and asymptomatic bradycardia (6 vs $1 \%$; $p<0.001)$ were noted to be more prevalent, this necessitated drug withdrawal in only $1 \%$ of the total cohort. The smallest study $(n=81)^{21}$ highlighted a significant increase in adverse events (64 vs 29\%; $p=0.004$ ), with phosphenes most strongly implicated. Symptomatic resolution occurred in all cases following study completion, but notably, there was no specific reporting of bradycardic events. 


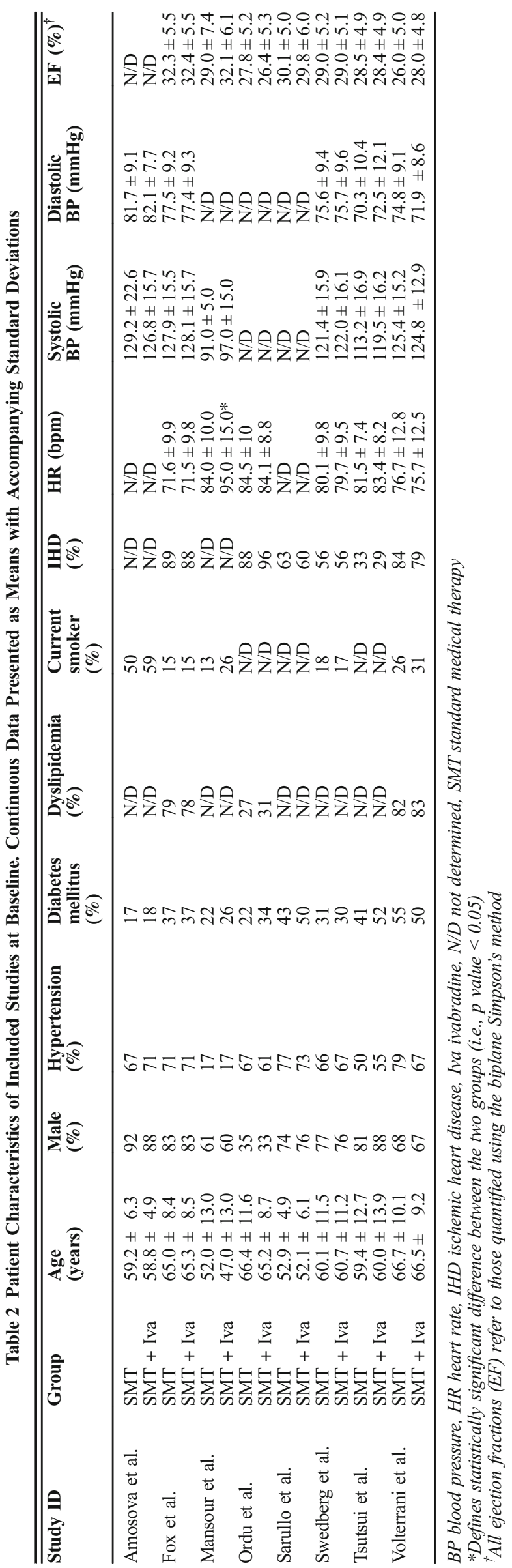

\section{Investigation of Heterogeneity}

Analyses for change in HR were stratified according to baseline LVEF, with distinction of two subgroups based on studies that included participants with LVEF $\leq 35$ or $>35 \%$ (albeit nonexclusively). One study was omitted as baseline EF was only described qualitatively. ${ }^{22}$ Results were consistent (Fig. 6a), with MD of $12.4 \mathrm{bpm}$ for studies with LVEF $\leq 35 \%$ (95\% CI 10.3$14.6 ; p<0.001)$ and $9.6 \mathrm{bpm}$ for LVEF $>35 \%$ (95\% CI 5.4 $13.8 ; p<0.001)$. There was moderate heterogeneity between subgroups $\left(I^{2}=27 \% ; p=0.24\right)$. Stratified analyses of change in EF were also comparable (Fig. 6b), with MD of 3.9\% for LVEF $\leq 35 \%(95 \%$ CI $3.2-4.6 ; p<0.001)$ and $3.5 \%$ for LVEF $>35 \%$ (95\% CI $1.2-5.7 ; p=0.003)$. There was no apparent heterogeneity between subgroups $\left(I^{2}=0 \% ; p=0.73\right)$.

\section{DISCUSSION}

\section{Summary of Findings}

This study is one of only two meta-analyses to assess effectiveness of ivabradine on hemodynamic and functional outcomes in LVSD. Adjunct therapy is associated with HR reduction, preservation of systolic BP, and a modest increase in EF. Despite substantial statistical heterogeneity, intervention effect measures were broadly preserved in sensitivity analyses and in subgroups stratified by baseline LVEF. Moreover, treatment was associated with a trend towards reduction in NT-proBNP levels. There was a marginal improvement in exercise capacity of unclear clinical relevance, but nonetheless, this correlated with broad improvement of other functional outcomes including peak oxygen consumption, NYHA class, and QoL. Ivabradine appears to be generally welltolerated with an adequate safety profile.

\section{Effects on Hemodynamics}

The two largest trials included in this analysis are heterogeneous with regard to patient characteristics and inclusion criteria. ${ }^{11,17}$ However, a pooled post hoc analysis of individual patient data from these two studies showed ivabradine to be associated with reductions in major outcomes including hospitalization and mortality. ${ }^{23}$ Results from our metaanalysis infer that conferred benefits may relate to a favorable hemodynamic profile. Indeed, the adverse effects of elevated HR in the context of LVSD are established and multi-factorial, relating to pathological ventricular remodeling, endothelial dysfunction, and accelerated atherogenesis. ${ }^{24}$

A separate meta-analysis in 2017 also suggests HR reduction from baseline with ivabradine treatment, though magnitude was less pronounced at $6.1 \mathrm{bpm}(95 \%$ CI $3.8-8.5$; $p<0.001){ }^{25}$ This disparity may primarily relate to inclusion of longer follow-up periods, with selection of 24 months $^{17}$ and 12 months $^{11}$ for the two largest trials. In addition, a more stringent inclusion criteria was applied with eligibility defined by $\mathrm{EF}<40 \%$. Pertinent data has also emerged from a 
Table 3 Pooled, Weighted Baseline Characteristics. Data Are Presented as Means, Unless Otherwise Stated

\begin{tabular}{llll}
\hline \hline & SMT $(\boldsymbol{n}=\mathbf{8 8 9 5})$ & $\begin{array}{l}\text { SMT + ivabradine } \\
(\boldsymbol{n}=\mathbf{8 9 2 8})\end{array}$ & $\boldsymbol{p}$ value \\
\hline Age (years) & 63 & 63 & 0.76 \\
Male (\%) & 80 & 80 & 0.99 \\
Hypertension (\%) & 69 & 69 & 0.99 \\
Diabetes mellitus (\%) & 35 & 35 & 0.99 \\
Dyslipidemia (\%) & 79 & 78 & 0.18 \\
Current smoker (\%) & 16 & 16 & 0.99 \\
IHD (\%) & 76 & 76 & 0.99 \\
HR (bpm) & 75 & 74 & 0.84 \\
Systolic BP (mmHg) & 125 & 126 & 0.86 \\
Diastolic BP (mmHg) & 77 & 77 & 0.96 \\
EF (\%) & 31 & 31 & 0.99 \\
\hline
\end{tabular}

$B P$ blood pressure, EF ejection fraction, HR heart rate, IHD ischemic heart disease, SMT standard medical therapy

retrospective SHIFT subgroup analysis, which has shown that benefits of ivabradine are most pronounced in those with highest baseline HR ( $\geq 75 \mathrm{bpm}) .{ }^{26}$ Moreover, a further substudy has shown that magnitude of HR reduction, rather than background $\mathrm{BB}$ dose per se, has primary influence on improved outcomes. ${ }^{27}$ In our study, there were significant variations in BB dosing and uptitration regimens which prohibited further subgroup analyses from being conducted.

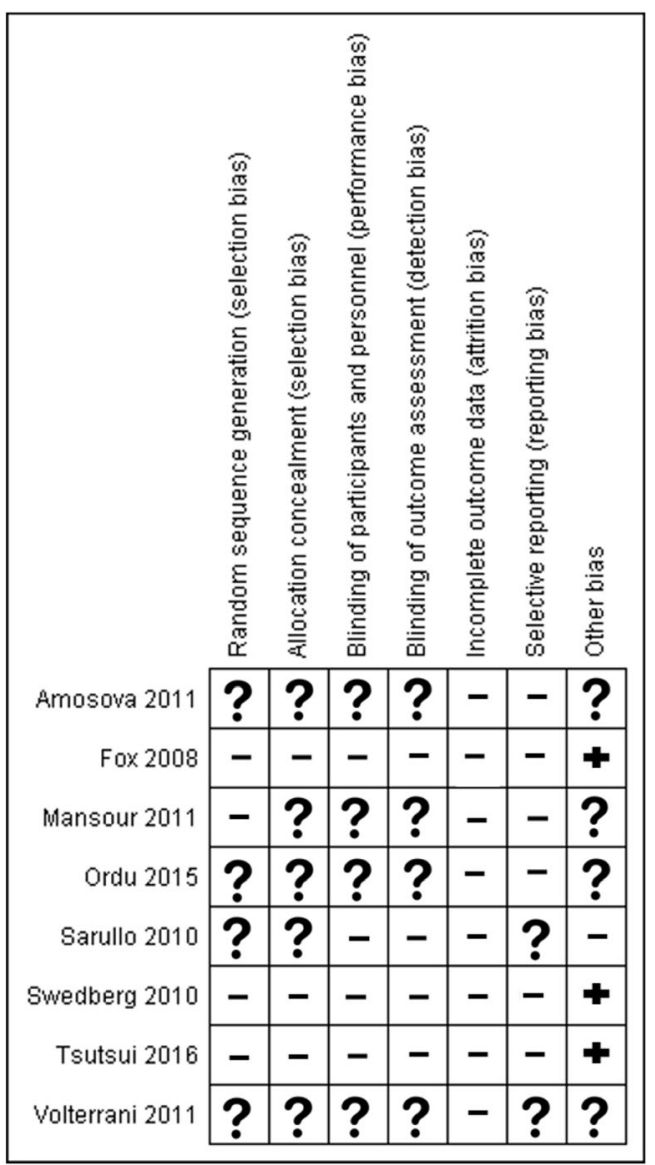

Figure 2 Risk of bias summary. $[-]$ indicates low risk of bias; [?] indicates unclear risk of bias; $[+]$ indicates high risk of bias.
A modest improvement in EF with ivabradine therapy is observed, which is comparable to results derived from the aforementioned meta-analysis (MD 3.3\%; 95\% CI 0.4-6.1; $p=0.03$ ). However, the relative contribution of pharmacological inotropy compared to physiological compensation in response to HR reduction is unclear. Pertinently, improved EF was also observed in all studies included in the SMT arm of the meta-analysis though magnitude was less marked. The notion of augmented EF with ivabradine is corroborated by results from the SHIFT echocardiography substudy, which showed reversal of cardiac remodeling, reduced left ventricle endsystolic volume index (LVESVI), and improved EF. ${ }^{28}$ These changes appeared to be independent of baseline LVEF, underlying etiology or BB use. As cardiac remodeling is a significant contributor to pathogenesis and progression of LVSD, these findings have potential clinical implications.

\section{Effects on Functional Capacity}

Our analysis also uniquely demonstrates that ivabradine has beneficial impact on functional capacity in these cohorts. It is noteworthy that exercise tolerance and peak oxygen consumption were only assessed in a small number of studies, and potential confounding effects of cardiac rehabilitation on observed trends cannot be ignored. Nonetheless, results are encouraging when it is considered that peak oxygen consumption has been shown to correlate with prognosis in patients with LVSD. ${ }^{29}$ Improvements in functional capacity may be attributed to potentiated HR reduction, though in direct contrast to BB, ivabradine has also been shown to preserve exercise-induced coronary vasodilation and tissue perfusion in animal models. ${ }^{30}$

The previously conducted meta-analysis focused primarily on effects relating to morbidity and mortality rather than functional outcomes. Whilst exercise tolerance in the form of 6MWD was assessed, this present study has made a concerted effort to consider a wider range of functional parameters including peak oxygen consumption, NYHA functional class, and QoL. Though QoL is broadly subjective, it is a pertinent endpoint in patients with chronic disease. The benefits observed with ivabradine therapy are likely related to co-existent effects on exercise tolerance, and findings are consistent with a SHIFT substudy that observed improvements in health-related QoL using the disease-specific Kansas City Cardiomyopathy Questionnaire (KCCQ). ${ }^{31}$

\section{Ivabradine and Non-severe LVSD}

Of relevance, our data suggests that hemodynamic benefits with regard to HR and $\mathrm{EF}$ are observed irrespective of baseline LVEF, with equivalent improvements in those with $\mathrm{EF}<35 \%$ (traditionally defined as severe LVSD) and $>35 \%$. For assessments on change in EF, both comparison groups were relatively small $(n=81$ [LVEF $<35 \%], n=142$ [LVEF $>35 \%]$ ), but these preliminary findings are encouraging and advocate further research to establish whether patients with LVSD of mild or moderate severity sustain equivalent benefit. 


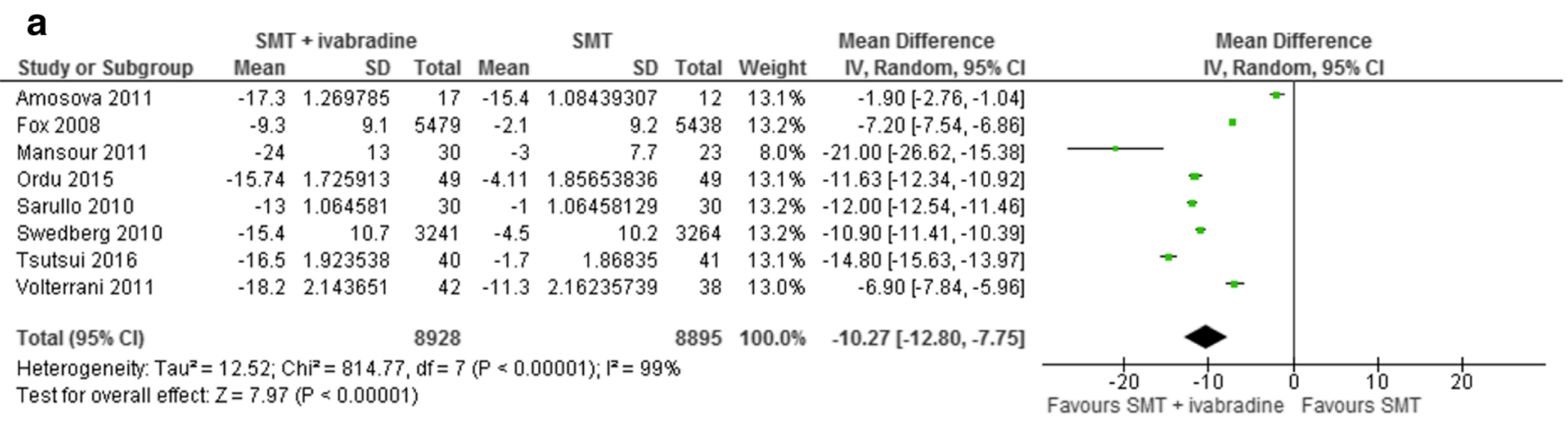

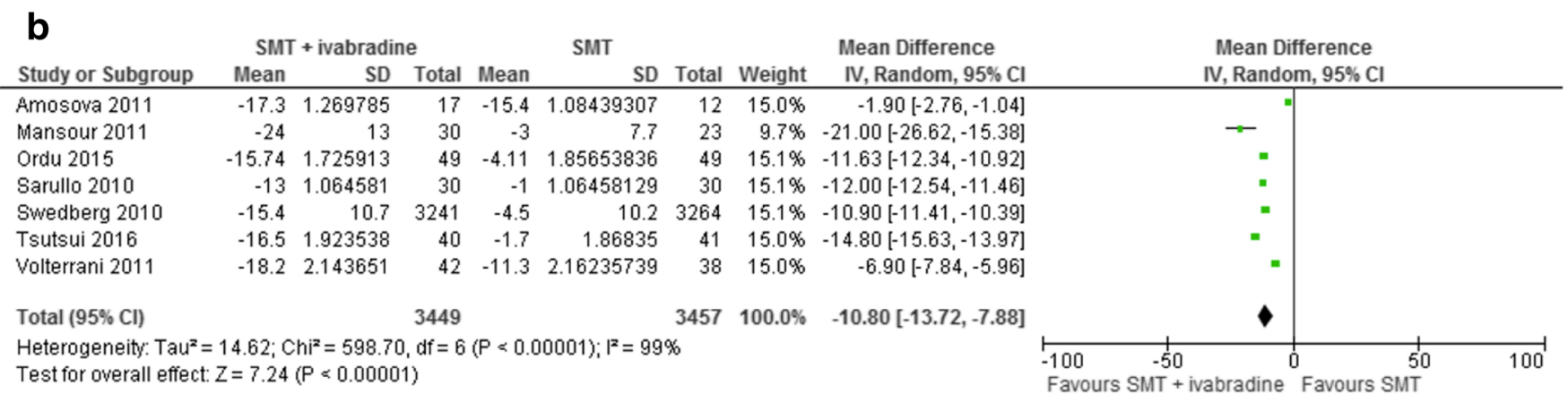

Figure 3 Forest plot for change in HR (a) and additional sensitivity analysis (b). Size of data markers correlate with the weight of each study. CI, confidence intervals (represented by horizontal bars); SD, standard deviation; SMT, standard medical therapy.

\section{Study Strengths}

The robustness of observed trends derived from our metaanalysis was enhanced by purposeful omission of nonrandomized cohort studies that are inherently prone to selection bias. Comparison of pooled, weighted characteristics, including traditional risk factors for LVSD and hemodynamic parameters, did not indicate significant baseline differences between the two groups. In addition, no geographical restrictions on study center were placed to provide a representative "real-world" perspective of patients with LVSD. Analysis of outcomes using an ITT approach was intended to minimize biased comparisons due to dropouts, though numbers lost or withdrawn were proportionately low in all studies included (data not shown).

\section{Study Limitations}

This meta-analysis does have inherent limitations. Post hoc analyses were excluded due to lack of access to patient level data and risk of individual participant overlap between studies, whilst original authors were not consulted to seek outstanding data. Only articles of English language were considered. Despite stringent inclusion criteria, inter-study variability existed

\begin{tabular}{|c|c|c|c|c|c|c|c|c|c|}
\hline \multirow{2}{*}{$\begin{array}{l}\text { A } \\
\text { Study or Subgroup }\end{array}$} & \multicolumn{3}{|c|}{ SMT + ivabradine } & \multicolumn{2}{|r|}{ SMT } & \multirow[b]{2}{*}{ Total } & \multirow[b]{2}{*}{ Weight } & \multirow{2}{*}{$\begin{array}{l}\text { Mean Difference } \\
\text { IV, Random, } 95 \% \mathrm{Cl}\end{array}$} & \multirow{2}{*}{$\begin{array}{l}\text { Mean Difference } \\
\text { IV, Random, } 95 \% \mathrm{Cl}\end{array}$} \\
\hline & Mean & SD & Total & Mean & SD & & & & \\
\hline Amosova 2011 & -1.2 & 5.232984 & 17 & -8.8 & 8.80818748 & 12 & $22.0 \%$ & $7.60[2.03,13.17]$ & -- \\
\hline Sarullo 2010 & -2 & 1.683251 & 30 & -2 & 2.59486673 & 30 & $39.1 \%$ & $0.00[-1.11,1.11]$ & | \\
\hline Volterrani 2011 & -5.3 & 2.287128 & 42 & -9.7 & 3.04341394 & 38 & $38.9 \%$ & $4.40[3.21,5.59]$ & $\square$ \\
\hline Total $(95 \% \mathrm{Cl})$ & & & 89 & & & 80 & $100.0 \%$ & $3.38[-0.49,7.26]$ & \\
\hline \multicolumn{9}{|c|}{$\begin{array}{l}\text { Heterogeneity: } \operatorname{Tau}^{2}=9.67 ; \mathrm{Chi}^{2}=31.92, \mathrm{df}=2(P \leq 0.00001) ;\left.\right|^{2}=94 \% \\
\text { Test for overall effect: } Z=1.71(P=0.09)\end{array}$} & 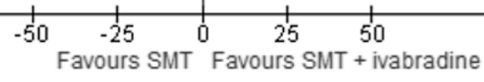 \\
\hline & \multicolumn{3}{|c|}{ SMT + ivabradine } & \multicolumn{2}{|r|}{ SMT } & & \multicolumn{2}{|c|}{ Mean Difference } & \multirow{2}{*}{$\begin{array}{l}\text { Mean Difference } \\
\text { IV, Random, } 95 \% \mathrm{Cl}\end{array}$} \\
\hline Study or Subgroup & Mean & SD & Total & Mean & SD & Total & Weight & IV, Random, 95\% Cl & \\
\hline Amosova 2011 & -1.8 & 2.799685 & 17 & -4.6 & 4.30164697 & 12 & $14.1 \%$ & $2.80[0.03,5.57]$ & F \\
\hline Volterrani 2011 & -1.7 & 1.735141 & 42 & -6.1 & 1.89854515 & 38 & $85.9 \%$ & $4.40[3.60,5.20]$ & \\
\hline Total $(95 \% \mathrm{Cl})$ & & & 59 & & & 50 & $100.0 \%$ & $4.17[3.08,5.27]$ & $i$ \\
\hline \multicolumn{9}{|c|}{$\begin{array}{l}\text { Heterogeneity: } \mathrm{Tau}^{2}=0.20 ; \mathrm{Chi}^{2}=1.18, \mathrm{df}=1(\mathrm{P}=0.28) ; \mathrm{I}^{2}=15 \% \\
\text { Test for overall effect: } Z=7.49(\mathrm{P}<0.00001)\end{array}$} & $\begin{array}{ccccc}1 & 1 & 1 & 1 \\
-100 & -50 & 0 & 50 & 100 \\
\text { Favours SMT } & \text { Favours SMT + iv }\end{array}$ \\
\hline
\end{tabular}

Figure 4 Forest plot for change in systolic BP (a) and diastolic BP (b). 


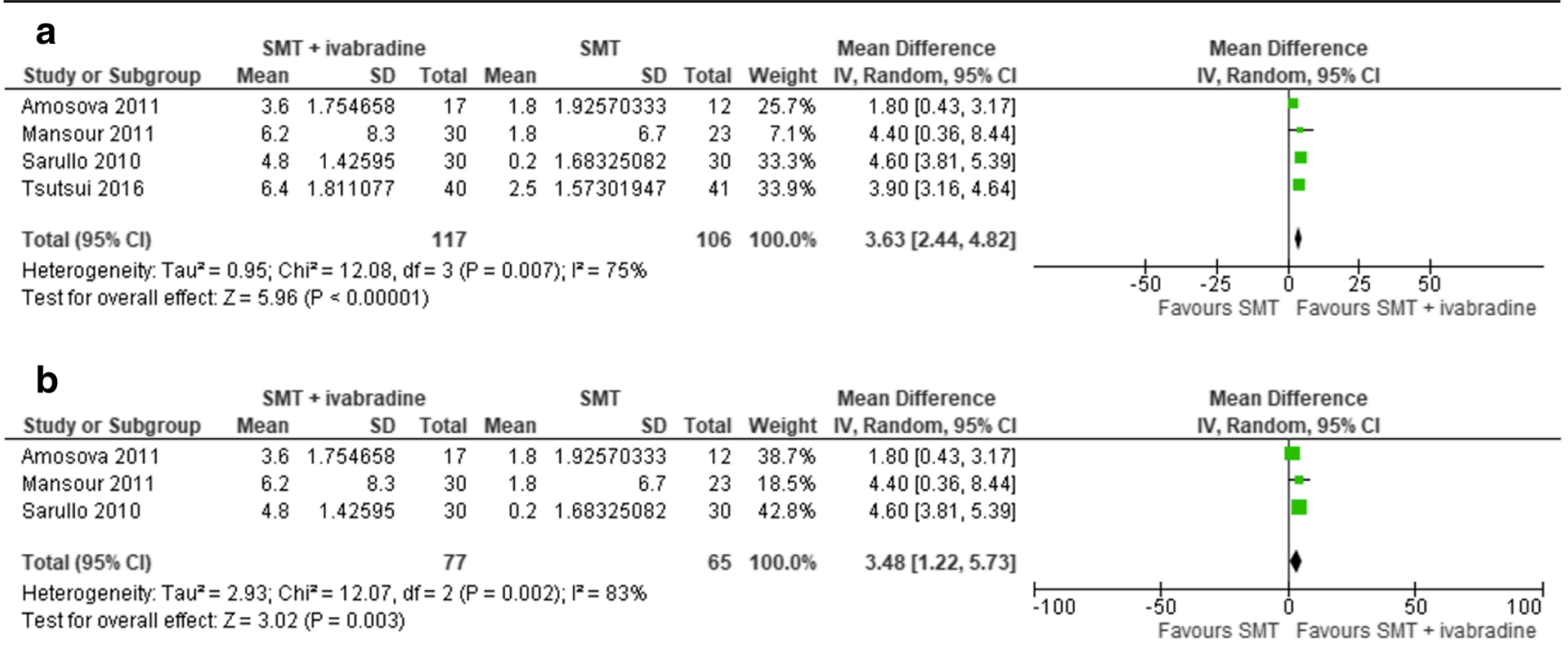

Figure 5 Forest plot for change in EF (a) and additional sensitivity analysis (b).

in baseline NYHA class and duration of SMT which may be confounding. Trial methodology was poorly reported for some studies, resulting in unclear risk of bias. Six of the eight studies had a total sample size of $<100$, which may provide

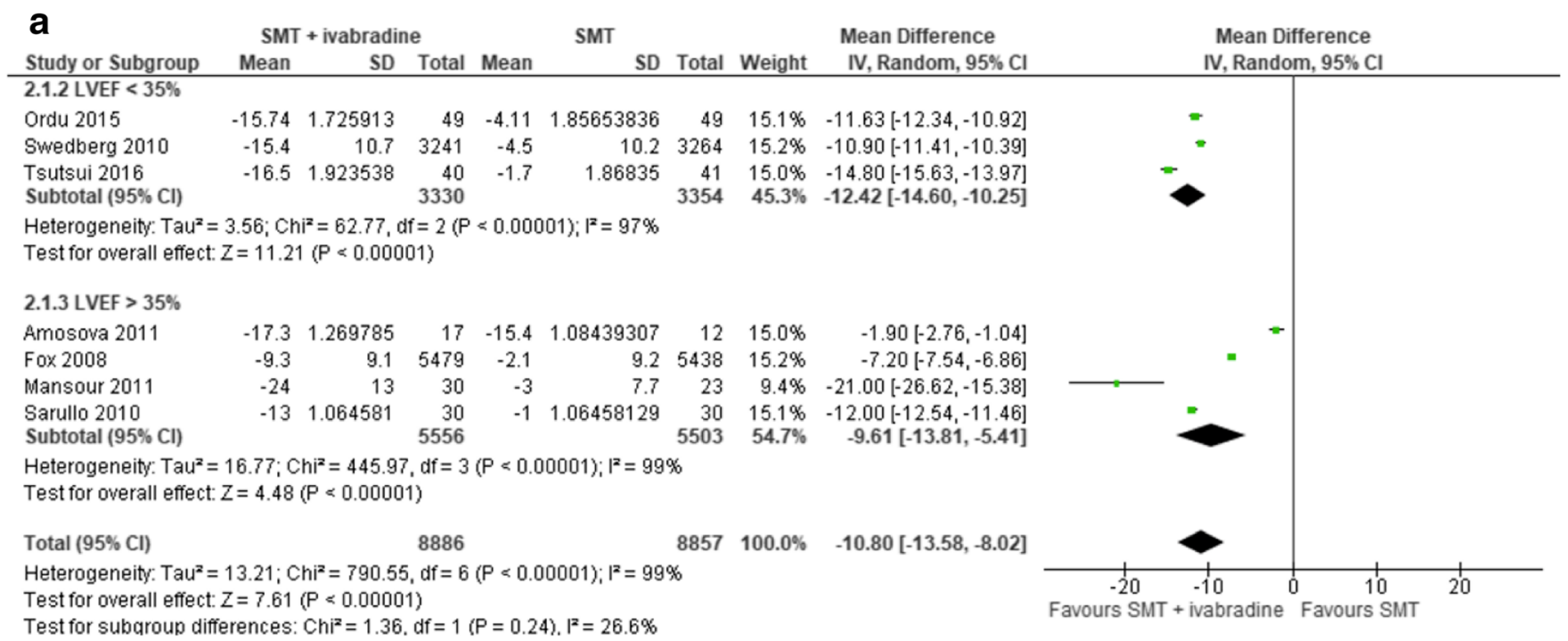

\section{b}

\begin{tabular}{|c|c|c|c|c|c|c|c|c|}
\hline Study or Subgroup & Mean & SD & Total & Mean & SD & Total & Weight & IV, Random, $95 \% \mathrm{Cl}$ \\
\hline \multicolumn{9}{|l|}{ 2.4.2 LVEF < $35 \%$} \\
\hline $\begin{array}{l}\text { Tsutsui } 2016 \\
\text { Subtotal }(95 \% \mathrm{Cl})\end{array}$ & 6.4 & 1.811077 & $\begin{array}{l}40 \\
40\end{array}$ & 2.5 & 1.57301947 & $\begin{array}{l}41 \\
41\end{array}$ & $\begin{array}{l}33.9 \% \\
33.9 \%\end{array}$ & $\begin{array}{l}3.90[3.16,4.64] \\
3.90[3.16,4.64]\end{array}$ \\
\hline \multicolumn{9}{|c|}{$\begin{array}{l}\text { Heterogeneity: Not applicable } \\
\text { Test for overall effect: } Z=10.34 \text { ( } P<0.00001)\end{array}$} \\
\hline \multicolumn{9}{|l|}{ 2.4.3 LVEF > 35\% } \\
\hline Amosova 2011 & 3.6 & 1.754658 & 17 & 1.8 & 1.92570333 & 12 & $25.7 \%$ & $1.80[0.43,3.17]$ \\
\hline Mansour 2011 & 6.2 & 8.3 & 30 & 1.8 & 6.7 & 23 & $7.1 \%$ & $4.40[0.36,8.44]$ \\
\hline $\begin{array}{l}\text { Sarullo } 2010 \\
\text { Subtotal }(95 \% \mathrm{Cl})\end{array}$ & 4.8 & 1.42595 & $\begin{array}{l}30 \\
77\end{array}$ & 0.2 & 1.68325082 & $\begin{array}{l}30 \\
65\end{array}$ & $\begin{array}{l}33.3 \% \\
66.1 \%\end{array}$ & $\begin{array}{l}4.60[3.81,5.39] \\
3.48[1.22,5.73]\end{array}$ \\
\hline
\end{tabular}

Heterogeneity: $\mathrm{Tau}^{2}=2.93 ; \mathrm{Chi}^{2}=12.07, \mathrm{df}=2(P=0.002) ; \mathrm{I}^{2}=83 \%$

Test for overall effect: $Z=3.02(P=0.003)$

Total $(95 \% \mathrm{Cl})$

117

$106 \quad 100.0 \%$

$3.63[2.44,4.82]$

Heterogeneity: Tau $^{2}=0.95 ; \mathrm{Chi}^{2}=12.08, \mathrm{df}=3(P=0.007) ; \mathrm{I}^{2}=75 \%$

Test for overall effect: $Z=5.96(P<0.00001)$

Test for subqroup differences: $C^{2}{ }^{2}=0.12, d f=1(P=0.73), I^{2}=0 \%$

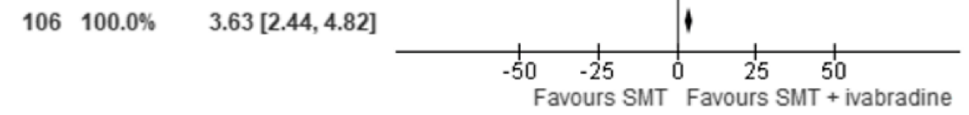

Figure 6 Forest plot for change in HR (a) and EF (b), stratified by baseline EF. 
insufficient power to detect true effects. Despite use of a random effects model, most outcomes were associated with substantial statistical heterogeneity. Lastly, only short-term follow-up data of around 3 months was available for assessed outcomes and it is therefore unclear whether trends translate in the longer term.

\section{CONCLUSION}

Amongst patients with LVSD, concurrent therapy with ivabradine results in significant reduction in resting HR without a drop in systolic BP, and improved EF. These changes appear to be coupled with improvements in objective and subjective functional capacity. The hemodynamic benefits of ivabradine extend to cohorts without severe LVSD. This suggests that further, large-scale RCTs are warranted in patients with LVSD of varying severity to scrutinize the effects of ivabradine on physiological parameters, symptom burden, and prognosis.

Acknowledgments: There are no internal or external sources of funding to declare. The corresponding author (PAP) has full access to all of the study data and final responsibility for the decision to submit for publication. This paper has not been presented in oral or poster format at any prior conferences.

Corresponding Author: Peysh A. Patel, MA; Department of Cardiol ogy, Leeds General Infirmary, Leeds, UK (e-mail: peysh@doctors.org. $u k)$.

Authors' Contribution PAP conceived the study design. AR and SP were involved with acquisition of data. PAP and NA acquired and analyzed data and drafted the manuscript as joint first authors. RMC, MTK, and KKW revised the paper critically for important intellectual content. All authors who contributed significantly to the work in this manuscript are listed.

\section{Compliance with ethical standards:}

Conflict of interest: The authors declare that they do not have a conflict of interest.

\section{REFERENCES}

1. Roger, VL. Epidemiology of heart failure. Circ Res 2013; 113:646-659.

2. Jhund PS, Macintyre $\mathbf{K}$, Simpson $\mathbf{C R}$, et al. Long-term trends in first hospitalization for heart failure and subsequent survival between 1986 and 2003: a population study of 5.1 million people. Circulation. 2009;119:515-23.

3. CONSENSUS Trial Study Group. Effects of enalapril on mortality in severe congestive heart failure. Results of the Cooperative North Scandinavian Enalapril Survival Study (CONSENSUS). N Engl J Med. 1987;316:1429-35.

4. Pitt B, Zannad F, Remme WJ, et al. The effect of spironolactone on morbidity and mortality in patients with severe heart failure. Randomized Aldactone Evaluation Study Investigators. N Engl J Med. 1999;341:70917.

5. McMurray JJ, Packer M, Desai AS, et al. PARADIGM-HF Investigators and Committees. Angiotensin-neprilysin inhibition versus enalapril in heart failure. N Engl J Med. 2014;371:993-1004.

6. CIBIS-II Investigators and Committees. The Cardiac Insufficiency Bisoprolol Study II (CIBIS-II): a randomised trial. Lancet. 1999;353:9-13.

7. Flannery G, Gehrig-Mills R, Billah B, Krum H. Analysis of randomized controlled trials on the effect of magnitude of heart rate reduction on clinical outcomes in patients with systolic chronic heart failure receiving beta-blockers. Am J Cardiol. 2008;101:865-869.
8. Bui AL, Grau-Sepulveda MV, Hernandez AF, et al. Admission heart rate and in-hospital outcomes in patients hospitalized for heart failure in sinus rhythm and in atrial fibrillation. Am Heart J. 2013;165:567-574.

9. Laskey WK, Alomari I, Cox M, et al. Heart rate at hospital discharge in patients with heart failure is associated with mortality and rehospitalization. J Am Heart Assoc. 2015;4.pii:e001626.

10. Psotka MA, Teerlink JR. Ivabradine: role in the Chronic Heart Failure Armamentarium. Circulation. 2016;133:2066-75.

11. Swedberg $\mathbf{K}$, Komajda $\mathbf{M}$, Böhm $\mathbf{M}$, et al. SHIFT investigators. Ivabradine and outcomes in chronic heart failure (SHIFT): a randomised placebo-controlled study. Lancet. 2010;376:875-85.

12. Yancy CW, Jessup M, Bozkurt B, et al. 2016 ACC/AHA/HFSA focused update on new pharmacological therapy for heart failure: an update of the 2013 ACCF/AHA Guideline for the Management of Heart Failure: a report of the American College of Cardiology/American Heart Association Task Force on Clinical Practice Guidelines and the Heart Failure Society of America. J Am Coll Cardiol. 2016;68:1476-1488.

13. Ponikowski P, Voors AA, Anker SD, et al. 2016 ESC Guidelines for the diagnosis and treatment of acute and chronic heart failure: The Task Force for the diagnosis and treatment of acute and chronic heart failure of the European Society of Cardiology (ESC). Developed with the special contribution of the Heart Failure Association (HFA) of the ESC. Eur Heart J. 2016;37:2129-2200.

14. Higgins JPT, Altman DG, Sterne JAC. Chapter 8: assessing risk of bias in included studies. In: Higgins JPT, Green S, eds. Cochrane Handbook for Systematic Reviews of Interventions version 5.1.0. Cochrane Collaboration, 2011.

15. Higgins JPT. Cochrane Handbook for Systematic Reviews of Interventions, 2011. The Cochrane Collaboration, 2011. http://www.cochranehandbookorg20112011 (Version 5.1.0).

16. Amosova E, Andrejev E, Zaderey I, Rudenko U, Ceconi C, Ferrari R. Efficacy of ivabradine in combination with beta-blocker versus uptitration of beta-blocker in patients with stable angina. Cardiovasc Drugs Ther. 2011;25:531-7.

17. Fox K, Ford I, Steg PG, Tendera M, Ferrari R. BEAUTIFUL investigators. Ivabradine for patients with stable coronary artery disease and left-ventricular systolic dysfunction (BEAUTIFUL): a randomised, doubleblind, placebo-controlled trial. Lancet. 2008;372:807-16.

18. Mansour S, Youssef A, Rayan M, Saleh MA. Efficacy of ivabradine in idiopathic dilated cardiomyopathy patients with chronic heart failure. Egyptian Heart Journal 2011;63:79-85.

19. Ordu S, Yildiz BS, Alihanoglu YI, et al. Effects of ivabradine therapy on heart failure biomarkers. Cardiol J. 2015;22:501-9.

20. Sarullo FM, Fazio G, Puccio D, et al. Impact of "off-label" use of ivabradine on exercise capacity, gas exchange, functional class, quality of life, and neurohormonal modulation in patients with ischemic chronic heart failure. J Cardiovasc Pharmacol Ther. 2010;15:349-55.

21. Tsutsui H, Momomura S, Yamashina A, et al. Heart rate control with If inhibitor, ivabradine, in Japanese patients with chronic heart failure-a randomized, double-blind, placebo-controlled phase II study. Circ J. 2016;80:668-76.

22. Volterrani M, Cice G, Caminiti G, et al. Effect of carvedilol, ivabradine or their combination on exercise capacity in patients with heart failure (the CARVIVA HF trial). Int J Cardiol. 2011;151:218-24.

23. Fox K, Komajda M, Ford I, et al. Effect of ivabradine in patients with left-ventricular systolic dysfunction: a pooled analysis of individual patient data from the BEAUTIFUL and SHIFT trials. Eur Heart J. 2013;34:2263-70.

24. Custodis F, Schirmer SH, Baumhakel M, Heusch G, Bohm M, Laufs U Vascular pathophysiology in response to increased heart rate. J Am Coll Cardiol. 2010;56: 1973-1983.

25. Narayanan MA, Reddy YNV, Baskaran J, et al. Ivabradine in the treatment of systolic heart failure-a systematic review and metaanalysis. World J Cardio. 2017;9:182-190.

26. Böhm M, Borer J, Ford I, et al. Heart rate at baseline influences the effect of ivabradine on cardiovascular outcomes in chronic heart failure. Analysis from the SHIFT study. Clin Res Cardiol. 2012;102:11-22.

27. Swedberg K, Komajda M, Böhm M, et al. SHIFT Investigators. Effects on outcomes of heart rate reduction by ivabradine in patients with congestive heart failure: is there an influence of beta-blocker dose? Findings from the SHIFT (Systolic Heart failure treatment with the I(F) inhibitor ivabradine Trial) study. J Am Coll Cardiol. 2012;59:1938-45.

28. Tardif JC, O'Meara E, Komajda M, et al. Effects of selective heart rate reduction with ivabradine on left ventricular remodelling and function: results from the SHIFT echocardiographic substudy. Eur Heart J. 2011;32:2507-15. 
29. Belardinelli R, Georgiou D, Cianci G, Purcaro A. Randomised controlled trial of long-term moderate exercise training in chronic heart failure. Effects on functional capacity, quality of life, and clinical outcome. Circulation. 1999;99:1173-1182.

30. Colin P, Ghaleh B, Monnet X, Hittinger L, Berdeaux A. Effects of graded heart rate reduction with ivabradine on myocardial oxygen consumption and diastolic time in exercising dogs. J Pharmacol Exp Ther. 2004;308:236-40.

31. Ekman I, Chassany O, Komajda M, et al. Heart rate reduction with ivabradine and health related quality of life in patients with chronic heart failure: results from the SHIFT study. Eur Heart J. 2011;32:2395-404. 